\title{
ESTRUCTURA DE BOSQUES SECUNDARIOS Y RASTROJOS MONTANO BAJOS DEL NORTE DE ANTIOQUIA, COLOMBIA
}

\author{
Structure on lower montane secondary forests and shrublands in northern \\ Antioquia, Colombia
}

Palabras clave: árboles muertos en pie, biomasa aérea, biomasa de lianas, biomasa subterránea, distribuciones diamétricas, modelo Hugershoff.

Key words: standing dead tree, above-ground biomass, liana biomass, below-ground biomass, diametric distribution, $\mathrm{Hu}$ gershoff model.

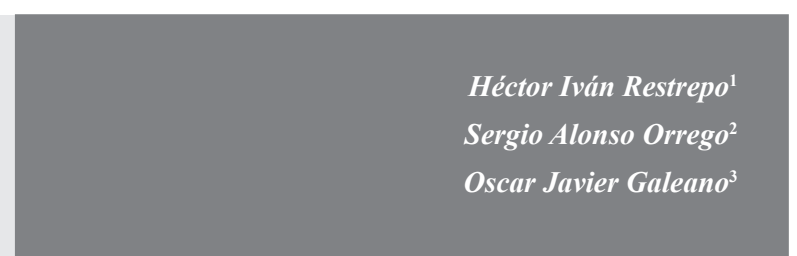

\section{RESUMEN}

Los bosques tropicales de montaña son considerados como los ecosistemas más biodiversos del mundo. Sin embargo, están siendo seriamente amenazados por la deforestación, la degradación y el cambio climático. Adicionalmente, su conservación también está en riesgo debido a que se conoce muy poco de su ecología. El objetivo de la presente investigación fue estudiar la estructura de fragmentos de bosque en el norte de Antioquia, para esto se establecieron cuatro parcelas permanentes en dos estados sucesionales; bosques secundarios y rastrojos. Para cada cobertura se analizó su distribución diamétrica por medio de modelos no lineales tipo Hugershoff. Asimismo, se estimó la biomasa aérea y subterránea de árboles y la biomasa aérea de lianas, así como la necromasa contenida en árboles muertos en pie. Todas las distribuciones diamétricas encontradas fueron en forma de $J$ invertida. Además, los modelos estimados explicaron satisfactoriamente el comportamiento de la estructura de los dos estados sucesionales analizados. La biomasa aérea y subterránea para los bosques secundarios fueron 91.6 y $76.3 \mathrm{t} \mathrm{ha}^{-1}$, respectivamente, mientras que para los rastrojos fueron 5.5 y $14.4 \mathrm{t} \mathrm{ha}^{-1}$. Por otra parte, la necromasa de bosques correspondiente a los árboles muertos en pie se estimó en $2.2 \mathrm{t} \mathrm{ha}^{-1}$.

\begin{abstract}
Tropical montane forests have the highest biodiversity in the world. However, they are being seriously threatened by deforestation, degradation and climate change. Their conservation is also at risk because we know little about their ecology. We established four permanent plots at two successional stages: secondary forest and cultivated areas. Distribution by diameter was analyzed using Hugershoff non-linear models. We estimated biomass of trees above and below-ground along with liana, under bush, understory and epiphyte biomass. In addition we measured necromass of standing dead trees, litter from wood and litterfall. We estimated total carbon in living and dead organic matter and in the soil. All the diametric distributions were of a $J$-inverted shape. The Hugershoff models successfully explained the behavior of the secondary forest and shrub structure. The biomass, necromass and total carbon were estimated at 185.7 and $29.8 \mathrm{t}$ $\mathrm{ha}^{-1}, 9.2$ and $3.8 \mathrm{t} \mathrm{ha} \mathrm{h}^{-1}$ and 151.7 and $78.2 \mathrm{t} \mathrm{ha}^{-1}$ respectively for forests and cultivated areas. An interesting finding is the high biomass of trees below-ground, lianas and epiphytes in tropical montane forest. Tropical montane forests provide important environmental services and therefore we have to search for better ways to conserve them.
\end{abstract}

\footnotetext{
Grupo de investigación en Bosques y Cambio Climático, Universidad Nacional de Colombia, sede Medellín. Calle 59A 63-020, Bloque 41, oficina 105, Medellín, Colombia. hirestrepoo@unal.edu.co. Autor para correspondencia.

Departamento de Ciencias Forestales, Universidad Nacional de Colombia, sede Medellín. Medellín, Colombia. saorrego@unal.edu.co

Maestría en Medio Ambiente y Desarrollo, Universidad Nacional de Colombia, sede Medellín. Medellín, Colombia. ojgaleanot@unal.edu.co
} 


\section{INTRODUCCIÓN}

Los bosques tropicales de montaña son los ecosistemas más biodiversos del mundo (Myers et al., 2000) debido a la gran variedad de condiciones climáticas, orográficas y edáficas (Richter, 2008). De estos ecosistemas, los Andes tropicales tienen la mayor diversidad con 30 000, 1728, 1095, 610, 595 y 380 especies de plantas, aves, anfibios, reptiles, mamíferos y peces de agua dulce, respectivamente (Mittermeier et al., 2011). El endemismo de la zona tropical andina se estima en 20000 especies de plantas y 1567 especies de animales vertebrados, las cuales son superiores al de todos los otros ecosistemas definidos como más biodiversos, pero también amenazados en el mundo (hotspots; Myers et al., 2000). Además, los patrones de distribución del endemismo de especies, a lo largo de gradientes altitudinales, muestran que se alcanza su máximo por encima de $2000 \mathrm{~m}$ de altitud (Kessler \& Kluge, 2008). Por todas estas razones, se considera que los bosques montanos tropicales tienen la mayor importancia en el mundo (Richter, 2008) y la más alta prioridad para la conservación, debido a que enfrentan una significativa amenaza antrópica (Newton, 2007).

La tasa de deforestación anual de los bosques tropicales montanos a nivel mundial se estima en $1.1 \%$ (Daumenge et al., 1995), la cual es casi el doble de la registrada para los bosques húmedos tropicales de tierras bajas (Achard et al., 2002). En Colombia, la tasa media de deforestación de los bosques por encima de $1000 \mathrm{~m}$ de altitud es del $0.63 \%$, aunque algunos municipios en esta franja altitudinal presentan tasas de conversión de bosques de $4.5 \%$ (Armenteras et al., 2011). La degradación de los bosques tropicales es otra importante amenaza, esta puede afectar $830 \mathrm{mil}$ ha año ${ }^{-1}$ en Latinoamérica (Achard et al., 2002). En este sentido, la extensión original de los bosques de montaña colombianos disminuyó aproximadamente $59 \%$ en el periodo $1989-1996$, por lo que es uno de los ecosistemas con mayor fragmentación en Colombia (Armenteras et al., 2003). Esta fragmentación puede ocasionar una disminución en la riqueza total de especies vegetales y en el número de individuos del sotobosque (Ochoa-Gaona et al., 2004), los cuales representan el relevo generacional del bosque. Por otra parte, los bosques andinos tropicales son extremadamente sensibles a los cambios climáticos, debido a las cortas distancias de migración que existen entre ellos y los bosques de tierras bajas (Bush et al., 2011). De esta forma, el riesgo de pérdida de especies por cambio climático podría ser sustancial si los cambios ocurren en corto tiempo y las especies no alcanzan a migrar a otra franja altitudinal.

Como resultado del proceso de regeneración natural, luego de una significativa reducción de la vegetación original, ocasionada ya sea por una perturbación natural o antrópica, aparecen los bosques secundarios (Akindele \& Onyekwelu, 2011). La estructura o composición de especies del dosel podría diferir si se compara con bosques primarios en sitios similares (Akindele \& Onyekwelu, 2011). Sin embargo, los bosques secundarios son de gran importancia, porque contribuyen a restaurar las características estructurales y funcionales de los bosques primarios (Guariguata \& Ostertag, 2001; Toledo et al., 2005), albergan la biodiversidad superviviente de los bosques primarios (Vilches et al., 2008; Chazdon et al., 2009), recuperan la fertilidad de los suelos (Fukushima et al., 2008), controlan la erosión y poseen características hidrológicas deseables (Chokkalingam et al., 2001); asimismo, son una importante fuente de madera de alta calidad y de leña (de las Salas, 2002) y presentan tasas positivas de acumulación de carbono (Chacón et al., 2007; Yepes et al., 2010).

Las áreas de montaña son consideradas una importante fuente de servicios ecosistémicos (Armenteras et al., 2011). Por su parte, los bosques tropicales de montaña presentan altas tasas de precipitación horizontal e interceptación y bajas tasas de evapotranspiración (Hölscher, 2008), esto los convierte en ecosistemas estratégicos para la conservación. Además, se ha encontrado que los primeros quince años de la sucesión se caracterizan por una rápida acumulación de biomasa aérea que puede exceder $100 \mathrm{t} \mathrm{ha}^{-1}$ (Brown \& Lugo, 1990). 
En este sentido, las áreas de agricultura con rentabilidad marginal ubicadas en regiones tropicales de montaña podrían destinarse para capturar carbono atmosférico en bosques secundarios (Fehse et al., 2002). Dicha estrategia de mitigación podría ser especialmente importante en países en vía de desarrollo, en los cuales se ha encontrado que los costos de captura de carbono son menores que los de países industrializados (Richards \& Stokes, 2004); esto debido a las diferencias entre las tasas de crecimiento de especies forestales y al costo de oportunidad de la tierra.

De igual forma, el establecimiento y monitoreo de parcelas permanentes es fundamental para estudiar procesos ecológicos que se suelen manifestar en lapsos de tiempo relativamente largos. Específicamente, el monitoreo sistemático y periódico de parcelas permanentes permite asegurar estudios de largo plazo de crecimiento diamétrico y de biomasa a nivel de individuos y parcelas. Las parcelas permanentes son muy útiles también en la identificación de cambios espaciales y temporales de la vegetación; ello permite el monitoreo de importantes procesos ecológicos relacionados con la composición florística, la estructura, el crecimiento, la mortalidad y el reclutamiento. Los inventarios a largo plazo pueden proveer información necesaria para definir prioridades de conservación y sirven como base para futuros trabajos.

El objetivo de la presente investigación fue caracterizar la estructura de algunos fragmentos de bosques y rastrojos del norte de Antioquia. Dicha caracterización comprende tanto la estructura horizontal, la cual incluye la determinación de las tallas de los individuos y las distribuciones diamétricas, como la cuantificación de las existencias de biomasa arbórea aérea y subterránea, la biomasa de lianas y la necromasa contenida en los árboles muertos en pie. Asimismo, se compararon los resultados encontrados entre los bosques secundarios y los rastrojos, así como con otras investigaciones realizadas en regiones de montaña tropicales.

\section{MATERIALES Y MÉTODOS}

\section{ÁREA DE ESTUDIO}

El área de estudio de la investigación se encuentra ubicada en la parte alta de la cuenca El Hato, norte de Antioquia, en jurisdicción del municipio de San Pedro. Los sitios en que se establecieron las parcelas permanentes tienen coordenadas promedias $838000 \mathrm{mE}$ y $1198000 \mathrm{mN}$, con una elevación en el rango 2550-2600 $\mathrm{m}$ de altitud.

\section{ESTABLECIMIENTO DE PARCELAS PERMANENTES}

Dos parcelas permanentes de $1000 \mathrm{~m}^{2}$ (0.1 ha) se establecieron en bosques secundarios avanzados en la parte alta de la cuenca El Hato, municipio de San Pedro. Se seleccionaron parcelas rectangulares (50 x $20 \mathrm{~m}$ ), en los límites del área de muestreo, para facilitar la identificación de aquellos árboles que estaban ubicados dentro de la parcela. Además, el establecimiento de parcelas de forma rectangular es mucho más expedito en terrenos con pendientes altas, si se compara con parcelas de forma circular. Aunque en las parcelas rectangulares es posible cometer errores de ángulos en sus vértices, los cuales ocasionan diferencias de áreas, en parcelas circulares, el radio se incrementa proporcionalmente al incremento del área, como también se incrementan los árboles en el borde de la parcela (Kangas, 2006).

El trazado de la parcela consistió en la selección de un vértice y el establecimiento de un lado de la parcela con brújula, hipsómetro y lienza. Luego se aplicaron los conceptos de rumbo y contrarrumbo para la definición de los demás lados. Se usó hilo de polipropileno de color amarillo para delimitar la parcela e identificar aquellas plantas leñosas que se encontraban dentro del rectángulo. En las parcelas se procedió a limpiar aquellos árboles con presencia de musgos o líquenes a una altura de $1.3 \mathrm{~m}$ sobre el suelo. A esta altura se midió el diámetro a la altura del pecho, o diámetro normal $D$ (o arriba de las bambas si las hubiera), de todas las plantas leñosas (árboles, arbustos, palmas y 
bejucos) con $D \geq 10 \mathrm{~cm}$. El instrumento para esta medición fue el calibrador forestal (forcípula), con exactitud de $0.5 \mathrm{~mm}$. Luego se marcaron con cruces de pintura amarilla los dos puntos de apoyo de las dos quijadas del calibrador forestal. Asimismo, en la tercera esquina de cada parcela se estableció una subparcela de 0.01 ha $(10 \times 10 \mathrm{~m})$. En ella se midieron con calibrador digital (pie de rey digital), con exactitud de $0.01 \mathrm{~mm}$, los diámetros de todas las plantas leñosas con $1 \leq D<10 \mathrm{~cm}$. Luego se marcaron con pintura amarilla los puntos de contacto de los brazos del calibrador con el tallo.

Todos los árboles se rotularon con pequeñas etiquetas en lámina de aluminio gravadas con su respectivo número, las cuales se colgaron de los clavos o de un collar holgado de alambre de cobre colocado alrededor del tallo en los árboles de la subparcela. También se pintó el número en el tallo de todos los árboles. En cada una de las parcelas se midieron las alturas totales $(H)$ de los árboles con un hipsómetro Vertex IV Haglöf, con resolución de $0.1 \mathrm{~m}$. En las subparcelas se midieron las alturas de todas las plantas leñosas pequeñas empleando flexómetro o hipsómetro Vertex.

El mismo protocolo se empleó para el establecimiento de parcelas permanentes en coberturas terrestres de rastrojos, caracterizados por la presencia de vegetación arbustiva de porte bajo junto con árboles dispersos de porte alto. El área de estas parcelas fue $500 \mathrm{~m}^{2}(25 \times 20 \mathrm{~m})$, en ella se midieron todas las plantas leñosas con $D \geq 5 \mathrm{~cm}$. Mientras que, en una subparcela de $5 \times 5 \mathrm{~m}$, se midieron los diámetros de todas las plantas leñosas con $1 \leq D$ $<5 \mathrm{~cm}$

En las parcelas de ambas coberturas se registraron otros elementos estructurales como la presencia y abundancia de lianas y epífitas vasculares y no vasculares, y se midieron los árboles muertos en pie.

\section{ESTRUCTURA}

La estructura se puede considerar como "cualquier situación estable o evolutiva, no anárquica, de una población o comunidad en la cual, aunque mínima, pueda detectarse algún tipo de organización representable por un modelo matemático, una ley estadística de distribución, una clasificación o un parámetro característico" (Rollet, 1980). Por tanto, el espectro de aplicación del concepto de estructura se amplía enormemente, incluso a la estructura de copas, estructura espacial de una especie, riqueza florística y diversidad (Rollet, 1980). Por ejemplo, algunos autores estudiaron elementos estructurales diferentes como la altura de los árboles (Ariza et al., 2009), el área basal (Álvarez-Yépiz et al., 2008), las copas de los árboles (Hiroaki \& McDowell, 2002; Aiba et al., 2004) y el índice de área foliar (Schwarzkopf et al., 2011). Sin embargo, la variable que más se usa para describir la estructura es el diámetro a la altura del pecho (Hering, 2003; Newton, 2007).

Adicionalmente, se calculó el promedio y la desviación estándar de $D, H$, área basal $(G)$, densidad o número de árboles por hectárea $(N)$ y el diámetro cuadrático medio $(D q)$. Se compararon las medias de cada variable por cobertura con el método diferencia mínima significativa DMS ( $t$-Student), usando un nivel de significancia $\alpha=0.05$.

Con los datos de $D$ se graficó el kernel de la función de densidad. Este método no paramétrico no requiere asumir una forma funcional específica para la distribución diamétrica. Por tanto, permite identificar tanto una forma general de distribución, como valores iniciales de los parámetros para la estimación de las funciones de distribución de probabilidades $(F D P)$. Un parámetro importante en la definición del kernel es el ancho de banda, el cual puede ser calculado como (Ecuación 1; Crawley, 2007),

$$
b=\frac{\max (x)-\min (x)}{2\left(1+\log _{2} n\right)} \quad \text { Ec. } 1
$$

donde, $x$ es el vector de diámetros a la altura del pecho y $n$ es el número de datos. Posteriormente, se estimaron los parámetros de las FDP gamma, Weibull, lognormal, logística, beta y exponencial 
negativa o exponencial, usando estimadores de máxima verosimilitud. Para cada distribución estimada, el ajuste estadístico se evaluó con una prueba de Kolmogorov-Smirnov.

Una especificación interesante de distribución diamétrica consiste en el modelo no lineal Hugershoff (Prodan, 1968; Cook et al., 1992), el cual es muy útil cuando no se obtienen ajustes satisfactorios con las formas más frecuentemente usadas de FDP. Los modelos Hugershoff y Weibull se suelen usar habitualmente para estudiar el crecimiento biológico, por su capacidad de representar bien el crecimiento tanto en estadios juveniles, como en los maduros (Cook et al., 1992). Además, ambas funciones son teóricamente más completas que otros modelos alternativos, los cuales presentan buen ajuste estadístico solo en la fase madura (Cook et al., 1992). La forma funcional del modelo Hugershoff es la ecuación 2

$$
f(x)=a x^{b} e^{-c x} \quad \text { Ec. } 2
$$

donde $f(x)$ es la frecuencia esperada, $x$ es la marca de clase y $a, b$ y $c$ son los parámetros a estimar. El procedimiento previo a la estimación fue dividir la muestra en clases diamétricas de igual tamaño y calcular el número de árboles por clase, con lo cual se obtuvo el par de datos $f(x)$ y $x$, donde $x=D$. Luego se calculó el modelo usando estimadores de máxima verosimitud y funciones de optimización no lineales.

Con las frecuencias por clase se realizó la prueba de bondad de ajuste $j i$ cuadrada $\left(\chi^{2}\right.$; Ecuación 3; Canavos, 1988)

$$
\chi^{2}=\sum_{i=1}^{k} \frac{\left.\left(f(x)_{i}-f \widehat{(x}\right)_{i}\right)^{2}}{f \widetilde{(x)_{i}}} \text { Ec. } 3
$$

donde $f(x)$ es frecuencia de los datos en la $i$-ésima clase y $f(x)_{i}$ es la frecuencia estimada para la $i$-ésima clase y $k$ es el número total de clases. Si el valor de $\chi^{2}$ es menor que un valor crítico $\chi_{(1-\alpha), k-1}^{2}$ , no se puede rechazar la hipótesis nula definida como (Canavos, 1988)

$$
H_{0}: F(x)=\widehat{F(x)}
$$

por tanto, $H_{0}$ se rechaza si para un valor observado de $x$, el valor de $\chi^{2}$ se halla en la región crítica de tamaño $\alpha$, correspondiente a un error tipo I (Canavos, 1988). En este sentido, se definió como aceptable un $\alpha=0.05$. Para todos los análisis estadísticos se usó el programa $\mathrm{R}_{\text {versión 2.11.1 }}$ ( $\mathrm{R}$ Development Core Team, 2010).

\section{ESTIMACIÓN DE BIOMASA Y NECROMASA}

Un elemento estructural especialmente importante es la biomasa. Esta se define como la cantidad total de materia orgánica viva sobre la superficie terrestre expresada en toneladas de materia seca por unidad de área (Brown, 1997). La biomasa es quizás la variable de mayor importancia no solo en estudios de productividad de ecosistemas, flujo de nutrientes y energía, sino también en el estudio de la contribución del cambio de los bosques, especialmente tropicales, al ciclo biogeoquímico del carbono (Parresol, 2002). La mayor cantidad de biomasa contenida en un bosque se encuentra almacenada en los productores primarios; es decir, en las plantas verdes fotosintéticas. La biomasa de las plantas vasculares mayores se suele dividir en biomasa aérea (i.e. tallos, ramas, hojas, flores y frutos) y biomasa subterránea (i.e. raíces; Schlegel et al., 2001).

La mayoría de las investigaciones relacionadas con la biomasa en bosques tropicales se han realizado en zonas de tierras bajas. Asimismo, son varios estudios han estimado las existencias de biomasa o carbono (Orrego \& del Valle, 2001; Cummings et al., 2002; Sierra et al., 2007; Quinto, 2010) y el crecimiento de la biomasa con el proceso sucesional (Uhl et al., 1988; Saldarriaga, 1994; Neeff \& dos Santos, 2005; del Valle et al., 2011). Estos últimos estudios permiten evaluar el comportamiento de la biomasa desde los estadios sucesionales más tempranos hasta que el bosque alcanza características estructurales similares a aquellas del bosque primario. Otros estudios intentan explicar el cambio de la biomasa en diferentes condiciones climáticas, edáficas y topográficas (Clark \& Clark, 2000; Homeier, 2008) y a lo largo de gradientes altitudinales (Leuschner \& Moser, 2008; Alves et 
al., 2010; Crausbay \& Hotchkiss, 2010; Culmsee et al., 2010; Girardin et al., 2010; Quinto, 2010).

En la tabla 1 se presentan las ecuaciones usadas para calcular los distintos componentes de biomasa y necromasa. Con datos de diámetros y adecuados factores de expansión, se calculó la biomasa aérea $(B a)$ y la biomasa de raíces gruesas (diámetro mayor a $5 \mathrm{~mm}$ ) (Brg) en cada una de las parcelas, al sumar las biomasas individuales de todos los árboles. La biomasa de las raíces finas $(B r f)$ (diámetro menor a $5 \mathrm{~mm}$ ) se calculó para toda la parcela usando
$G$ como variable independiente. Se compararon las medias de cada variable por cobertura con el método diferencia mínima significativa $D M S$ ( $t$-Student) utilizando un nivel de significancia $\alpha=$ 0.05 .

La biomasa de lianas $(B l)$ se calculó como el promedio de dos ecuaciones alométricas estimadas para bosques tropicales de tierras bajas y premontanos. No se encontró referencia alguna que permitiera estimar dicha biomasa para bosques montanos.

Tabla 1. Ecuaciones empleadas para el cálculo de distintos componentes de biomasa y necromasa. $D$ : diámetro normal $(\mathrm{cm}), G$ : área basal $\left(\mathrm{m}^{2} \mathrm{ha}^{-1}\right), f$ : factor de corrección que se definió arbitrariamente como 0.5

\begin{tabular}{|c|c|c|c|}
\hline Componente & Ecuación & Unidades & Fuente \\
\hline \multicolumn{4}{|l|}{ Biomasa } \\
\hline Arbórea aérea & $B a=0.073 D^{2.373}$ & $\mathrm{~kg}$ árbol $^{-1}$ & Álvarez et al. (2012) \\
\hline Arbórea de raíces gruesas & $B r g=0.151 \mathrm{D}^{1.899}$ & $\mathrm{~kg}$ árbol $^{-1}$ & Bolívar et al. (2006) \\
\hline Arbórea de raíces finas & $B r f=7+0.288 \cdot G$ & $\mathrm{t} \mathrm{ha}^{-1}$ & Sierra et al. (2001) \\
\hline Arbórea total & $B t=B a+B r g+B r f$ & $\mathrm{t} \mathrm{ha}^{-1}$ & \\
\hline Lianas & $B l=\left(1.07 \cdot D^{2.17}+1.03 \cdot D^{1.84}\right) / 2$ & kg liana ${ }^{-1}$ & $\begin{array}{l}\text { Gerwing \& Farias (2000), Sierra et al. } \\
\text { (2007) }\end{array}$ \\
\hline \multicolumn{4}{|l|}{ Necromasa } \\
\hline Árboles muertos en pie & $a m p=f \cdot 0.073 \mathrm{D}^{2.373}$ & $\mathrm{~kg}$ árbol $^{-1}$ & Álvarez et al. (2012) \\
\hline
\end{tabular}

Tabla 2. Resumen de las variables estructurales medidas y estimadas para todos los árboles con $D \geq 1 \mathrm{~cm}$ bosques secundarios y rastrojos del norte de Antioquia. Las ecuaciones para el cálculo de cada uno de los compartimientos de la masa total se pueden consultar en la tabla 1. Las letras mayúsculas en las variables indican la agregación por hectárea. El asterisco $\left(^{*}\right)$ representa una diferencia estadísticamente significativa por el método DMS ( $t$-Student) entre las coberturas $(\alpha=0.05)$

\begin{tabular}{|c|c|c|c|c|}
\hline \multirow{2}{*}{ Variables estructurales } & \multirow{2}{*}{ Unidad } & \multicolumn{3}{|c|}{ Cobertura } \\
\hline & & Bosque Secundario & Rastrojo & \\
\hline \multicolumn{5}{|l|}{ Dasométricas } \\
\hline Diámetro normal $(D)$ & $\mathrm{cm}$ & $5.75 \pm 4.64$ & $2.17 \pm 1.18$ & $*$ \\
\hline Altura $(H)$ & $\mathrm{m}$ & $6.79 \pm 3.4$ & $2.58 \pm 0.62$ & $*$ \\
\hline Área basal $(G)$ & $\mathrm{m}^{2} \mathrm{ha}^{-1}$ & $38.71 \pm 0.75$ & $3.7 \pm 1.54$ & $*$ \\
\hline Densidad $(N)$ & árb. ha-1 & $9030 \pm 580$ & $7730 \pm 580$ & \\
\hline Diámetro cuadrático medio $(D q)$ & $\mathrm{cm}$ & $7.39 \pm 0.26$ & $2.42 \pm 0.5$ & $*$ \\
\hline \multicolumn{5}{|l|}{ Biomasa } \\
\hline Arbórea aérea $(B a)$ & $\mathrm{t} \mathrm{ha}^{-1}$ & $91.6 \pm 1.2$ & $5.5 \pm 2.9$ & $*$ \\
\hline Arbórea raíces gruesas (Brg) & $\mathrm{tha}^{-1}$ & $58.2 \pm 5.87$ & $6.3 \pm 2.83$ & $*$ \\
\hline Arbórea de raíces finas (Brg) & $\mathrm{t} \mathrm{ha}^{-1}$ & $18.1 \pm 0.21$ & $8.1 \pm 0.5$ & * \\
\hline Lianas $(B l)$ & $\mathrm{tha}^{-1}$ & $5.7 \pm 1.98$ & & \\
\hline \multicolumn{5}{|l|}{ Necromasa } \\
\hline Árboles muertos en pie $(A m p)$ & $\mathrm{t} \mathrm{ha}^{-1}$ & $2.2 \pm 0.28$ & & \\
\hline
\end{tabular}


También se estimó la necromasa contenida en los árboles muertos en pie ( $A m p)$, la cual se calculó como la sumatoria de la estimación de la biomasa aérea individual $(B a)$ multiplicada por un factor de corrección que se definió arbitrariamente como 0.5. Dicha corrección se explica por la masa perdida de árboles tronchados y en la caída de ramas y hojas.

\section{RESULTADOS}

En la tabla 2 se presenta el resumen estadístico de las principales variables estructurales por cobertura. Las dimensiones promedias de $D, D q, H$ y $G$ son significativamente mayores en el bosque secundario que en rastrojos $(p<0.05)$. Sin embargo, no se encontró diferencia significativa en $N$ ( $p$ $=0.15$ ). También se encontró que $B a, B r g, B r f$ son significativamente diferentes entre las coberturas $(p<0.05)$.

Para la caracterización de la estructura diamétrica se estimaron los parámetros de las FDP Weibull, gamma, lognormal, logística, beta y exponencial. Con los parámetros estimados se evaluó el comportamiento de la $F D P$ respecto a los datos por medio de métodos gráficos y la prueba de bondad de ajuste Kolmogorov-Smirnov. Dichos análisis sugieren que los datos no se ajustan satisfactoriamente a ninguna de las $F D P$ estimadas para los dos tipos de coberturas $(p<0.05)$.

El procedimiento alternativo cuando se rechaza la hipótesis nula con las FDP clásicas es estimar un modelo de regresión, lineal o no lineal, que permita relacionar las frecuencias observadas con clases diamétricas. Para este análisis se eligió el modelo Hugershoff por su flexibilidad y características teóricas y matemáticas deseables (Cook et al., 1992). El modelo Hugershoff estimado para la cobertura de bosque secundario es la ecuación 5 .

$$
f(x)=11.102 \cdot \exp [-0,193 \cdot x] \quad \text { Ec. } 5
$$

donde $f(x)$ es la frecuencia absoluta (árb. ha ${ }^{-1}$ ) y $x$ es la marca de clase diamétrica $(\mathrm{cm})$. Todos los parámetros fueron estadísticamente significativos $(p<0.01)$. En un modelo estimado previamente se encontró que el parámetro $b$ no fue estadísticamente diferente de cero ( $p=0.34$ ), por tanto, se excluyó del modelo. Así, con base en la ecuación 2, si $b=0$ entonces $x^{0}=1$. En la figura 1 se aprecia el ajuste del modelo al histograma de frecuencias con ocho clases diamétricas. También se puede apreciar que el comportamiento de la curva tiene trayectoria similar al kernel de la función de densidad. De esta forma, la prueba de bondad de ajuste jicuadrada permite concluir que no existe diferencia significativa entre las frecuencias observadas y estimadas por clase diamétrica (Figura 1).

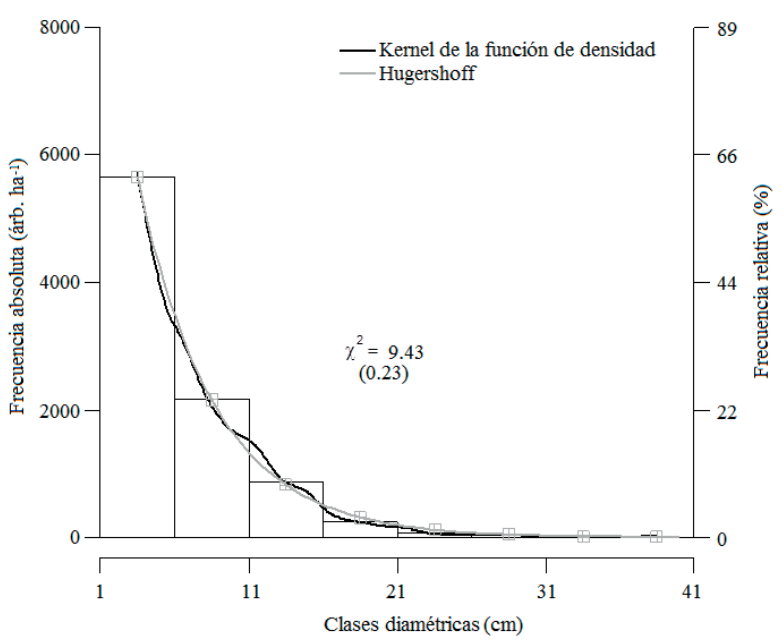

Figura 1. Histograma de frecuencias, modelo Hugershoff estimado y Kernel de la función de densidad para frecuencias absolutas por clase de diámetro para bosque secundario. El valor entre paréntesis corresponde al valor $p$ de la prueba $\chi^{2}$

Se encontró que los bosques tienen una distribución en forma de $\mathrm{J}$ invertida. En la primera clase diamétrica se encuentra el $63 \%$ de los árboles, mientras que en la última $0.06 \%$. El modelo Hugershoff estimado para determinar las frecuencias de árboles por clase diamétrica en rastrojos se expresa como la ecuación 6

$$
f(x)=29.843 \cdot \exp [-0,936 \cdot x] \text { Ec. } 6
$$

Todos los parámetros fueron muy significativos $(p<0.01)$. Al igual que en la cobertura de bosque secundario, el parámetro $b$ fue no significativamente diferente de cero $(p=0.47)$. Por tanto, se excluyó del modelo. La prueba de bondad de ajuste ji-cuadrada sugiere que no hay evidencia suficiente para concluir que existe diferencia entre 
las frecuencias observadas y estimadas, por esto se acepta la hipótesis nula (Figura 2). En la figura 2 se puede apreciar el histograma de frecuencias y el comportamiento de la función de densidad y del modelo estimado. La distribución de los datos es en forma de $\mathrm{J}$ invertida con $75 \%$ de los árboles en la primera clase diamétrica y $0.7 \%$ en la última. Asimismo, el modelo estimado para rastrojos tiene valores de parámetros que exceden a los del modelo estimado para bosques secundarios. El parámetro a representa una mayor densidad de árboles en las primeras clases de diámetro, mientras que el parámetro $b$ representa una mayor pendiente o mayor disminución de densidad de árboles a medida que el diámetro se incrementa.

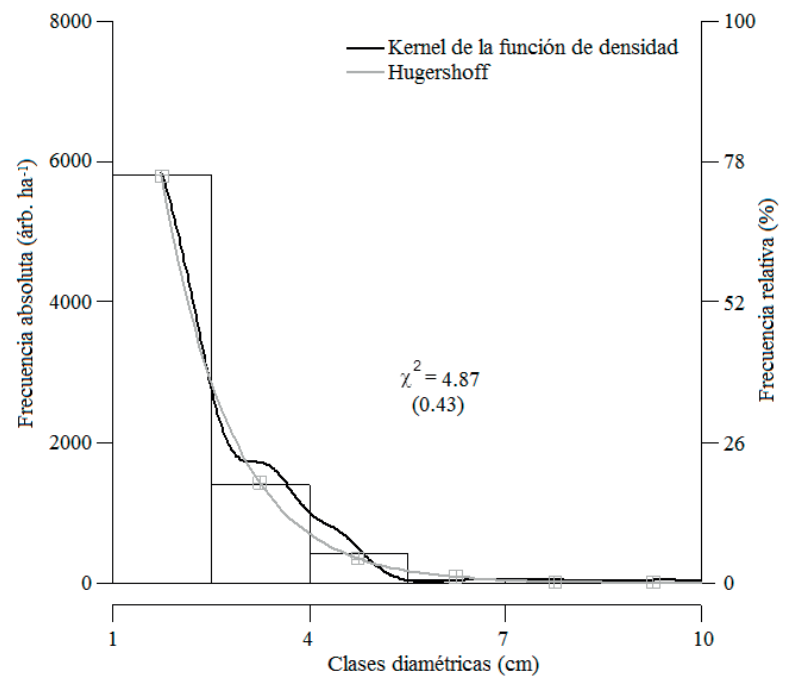

Figura 2. Histograma de frecuencias, modelo Hugershoff estimado y Kernel de la función de densidad para frecuencias absolutas por clase de diámetro para barbechos. El valor entre paréntesis corresponde al valor $p$ de la prueba $\chi^{2}$

En los bosques secundarios, la distribución de biomasa total $(B t=B a+B r g+B r f)$ por clases diamétricas es unimodal, con una participación de $57 \%$ de la biomasa en clases diamétricas intermedias $(6-11,11-16 \mathrm{~cm})$ y una menor participación de la biomasa en las clases diamétricas inferior y superior. Aunque la clase diamétrica inferior tiene muchos árboles, la suma de sus biomasas es muy baja debido a sus bajas tallas. Por el contrario, en clases diamétricas superiores los individuos tienen tallas mayores que representan mayor biomasa individual, pero con muy pocos individuos (Figura 3). Además, en la primera clase de diámetro, la biomasa subterránea $(B r g+B r f)$ es superior a $B a$. En la segunda clase diamétrica, la $B a$ y $B r g+B r f$ son aproximadamente iguales. Y, a partir de la tercera clase diamétrica, la $\mathrm{Ba}$ es superior a $\mathrm{Brg}+$ $\operatorname{Brf}$ (Figura 3).

La biomasa de los rastrojos se distribuye en forma similar a una $\mathrm{J}$ invertida, con $53 \%$ de la biomasa en las dos primeras clases de diámetro. En los rastrojos $B r g+B r f$ representa, en promedio, $75 \%$ de $B t$. En las tres primeras clases diamétricas, la $B a$ es aproximadamente igual a la $B r f$, mientras que en las últimas clases de diámetro, la proporción de $B r f$ es mayor y la $B a$ es similar a la Brg (Figura 4).

En los bosques, la relación entre $B a, B r g$ y $B r f$ respecto a $B t$ fue $55 \%, 35 \%$ y $10 \%$, respectivamente. Las mismas proporciones para los rastrojos fueron $28 \%, 32 \%$ y $41 \%$. Por otra parte, las relación $\mathrm{Brg} / \mathrm{Ba}$ y $(\mathrm{Brg}+\mathrm{Brf}) / \mathrm{Ba}$ fueron $64 \%$ y $83 \%$ para bosques, mientras que la mismas relaciones para rastrojos fueron $115 \%$ y $262 \%$. Dicho comportamiento sugiere que la asignación a dichos componentes varía con la cobertura analizada.

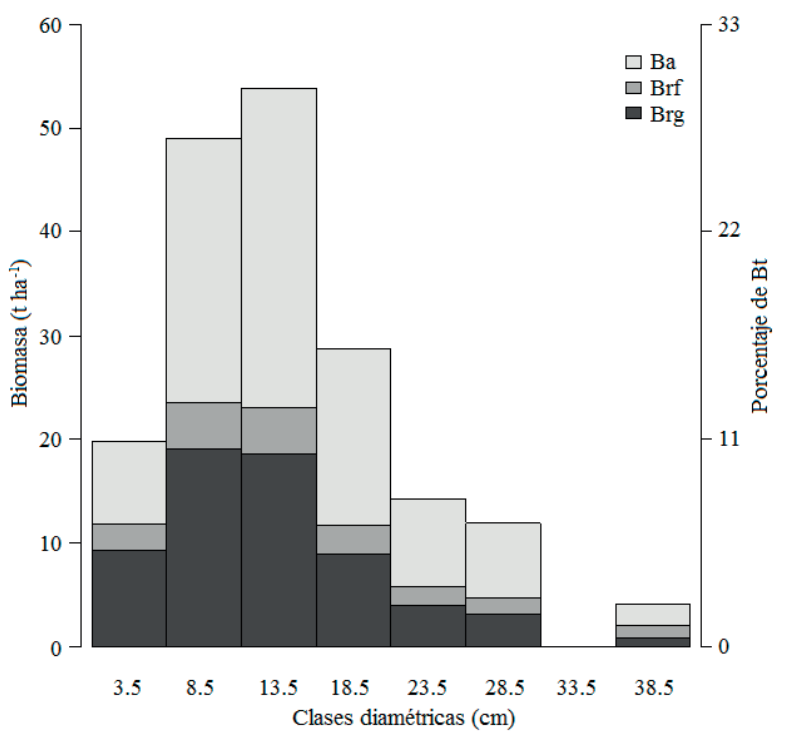

Figura 3. Distribución de la biomasa por clase diamétrica para los bosques secundarios. La frecuencia relativa es respecto a la biomasa total para la cobertura, igual a $168 \mathrm{t} \mathrm{ha}^{-1}$ 
Otros elementos importantes en la estructura de un bosque son la presencia de lianas (van der Heijden \& Phillips, 2008), epífitas (Newton, 2007) y árboles muertos en pie (Orrego \& del Valle, 2001). En los bosques secundarios se encontró que las lianas tienen una densidad de 700 lianas ha-1 y un área basal de $0.51 \mathrm{~m}^{2} \mathrm{ha}^{-1}$. Por otra parte, la densidad de epífitas encontrada se registró entre media-alta y la cantidad de árboles muertos en pie fue 310 árboles ha ${ }^{-1}$, que corresponde a $3.4 \%$ de los árboles vivos con una necromasa que corresponde a $2.4 \%$ de la $B a$. Un aspecto estructural importante y diferenciador entre bosques y rastrojos es que no se encontraron lianas, epífitas y árboles muertos en pie en los rastrojos.

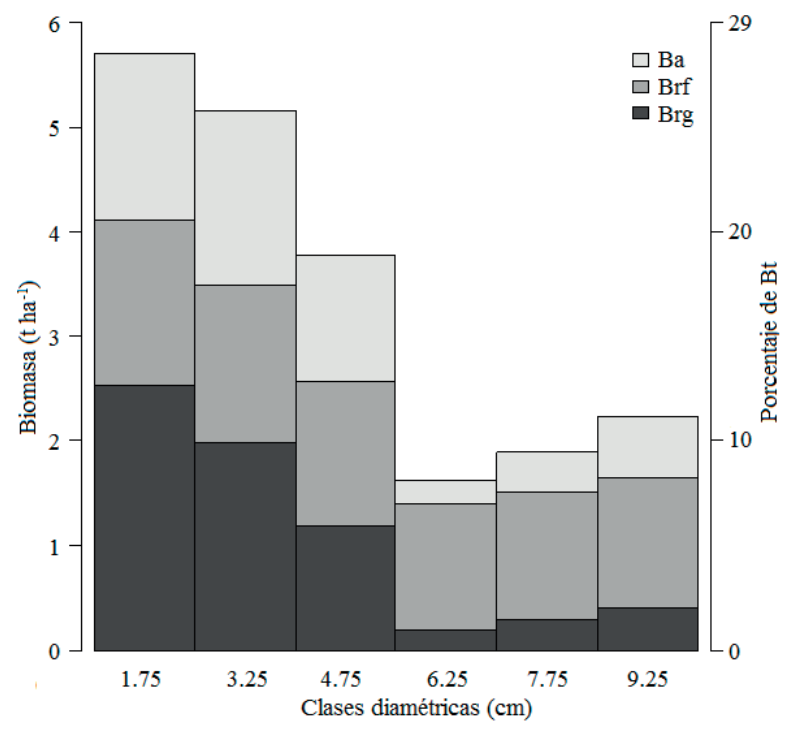

Figura 4. Distribución de la biomasa por clase diamétrica para los barbechos. La frecuencia relativa es respecto a la biomasa total para la cobertura, igual a 20 $\mathrm{t} \mathrm{ha}^{-1}$

\section{DISCUSIÓN}

Los elementos estructurales de los bosques y rastrojos del norte Antioquia estudiados en la presente investigación son similares a los encontrados en otros lugares de Colombia y del mundo (Tabla 3 ). Los otros estudios correspondieron a investigaciones en bosques montano bajos tropicales, con elementos estructurales comparables; sin embargo esta comparación, en algunos casos, es difícil debido a diferencias como: diámetro mínimo de muestreo, altura mínima medida, amplitud de clase y número clases diamétricas, etc. No obstante, la información presentada permitió la confrontación de todos los elementos estructurales estudiados en la presente investigación.

Las tallas promedio de los árboles con $D \geq 1 \mathrm{~cm}$ son inferiores a lo reportado por otros autores. Sin embargo, si se consideran individuos con $D \geq 10$, las variables estructurales tienden a ser similares a las encontradas en otros estudios. Así, el diámetro promedio y la altura promedia de los árboles con $D \geq 10 \mathrm{~cm}$ se encuentra en el rango reportado en la literatura. No obstante, en todos los casos, el diámetro máximo reportado por otros autores es superior al encontrado en la presente investigación.

El área basal también es inferior a la reportada por la mayoría de los autores tanto para los árboles con $D \geq 1 \mathrm{~cm}$, como para aquellos con $D \geq 10 \mathrm{~cm}$. Por el contrario, la densidad encontrada en la presente investigación es, en general, superior a la reportada por otros autores.

Las distribuciones diamétricas encontradas en la presente investigación fueron en forma de $J$ invertida, lo cual es consistente con lo hallado en estudios previos. Todos ellos presentan en sus distribuciones diamétricas e histogramas de frecuencia más de $50 \%$ y, en algunos casos, más de $80 \%$ de los árboles en la primera clase de diámetro. Este tipo de estructura dominada por muchos árboles pequeños sugiere que el rodal ha sufrido una reciente regeneración o establecimiento, después de un evento de disturbio (Newton, 2007). Por el contrario, la presencia de algunos árboles grandes y gran cantidad de árboles pequeños puede interpretarse como rodales maduros en los cuales ocurre un reclutamiento permanente de individuos nuevos (Newton, 2007). A medida que se incrementa el tamaño, disminuye la cantidad de árboles por unidad de área, esto configura una forma de $J$ invertida. Aunque se debe tener presente que no siempre la edad y el tamaño están estrechamente relacionados (Newton, 2007), la estructura disetánea, característica de este tipo de distribuciones diamétricas, es la mejor garantía de la supervivencia de la comunidad forestal, ya que 
los individuos de tallas superiores son eliminados ocasionalmente y sustituidos sin dificultad por individuos de las categorías diamétricas inferiores (Lamprecht, 1990).

Los componentes de biomasa analizados son, en general, similares a los reportados en casi todas las investigaciones encontradas (Tabla 3). Sin embargo, la $B a$ en todas las otras investigaciones es superior a la encontrada en el presente estudio. Igualmente, no se encontraron referencias de estudios en zona de montaña que permitieran comparar la $B a$ de los rastrojos. No obstante, la $B a$ de rastrojos está en el rango $5.4-17.1 \mathrm{t} \mathrm{ha}^{-1}$ en rastrojos de las franjas basal tropical y premontano de Porce (Orrego \& del Valle, 2001; del Valle et al., 2011). La Brg de los bosques fue $36.8 \mathrm{t} \mathrm{ha}^{-1}$, superior a la reportada por otros autores, la cual está entre 16.2 y $27.6 \mathrm{t} \mathrm{ha} \mathrm{h}^{-1}$. La Brf de los bosques se encuentra en el rango de otras investigaciones. $\mathrm{Al}$ igual que para la $B a$, no se encontró información que permitieran comparar $B r g$ y $B r f$ de los rastrojos. Para los bosques de Porce se encontró que $B r g$ y Brf fue 5 y $7 \mathrm{t} \mathrm{ha}^{-1}$, respectivamente (del Valle et al., 2011), valores similares a los encontrados en la presente investigación.

Se ha encontrado que con el aumento de la elevación las tallas medias de los individuos decrece (Whitmore, 1998). Para explicar este fenómeno se han sugerido múltiples hipótesis relacionadas con la temperatura, humedad, fertilidad, fuertes vientos, etcétera. Sin embargo, la consecuencia inmediata es el cambio en los patrones de asignación del carbono entre la biomasa aérea y la biomasa subterránea (Leuschner \& Moser, 2008; Quinto, 2010). La $B a$ presente en los bosques montanos es menor que la encontrada en bosques primarios y secundarios del piso basal o premontano, la cual se encuentra en promedio en el rango 180-400t ha $^{-1}$ (Leuschner \& Moser, 2008; Quinto, 2010). Por ejemplo, en la zona de Zamora-Chinchiape, en Ecuador, se encontró que a medida que se incrementa la elevación, también se aumenta la relación entre la biomasa subterránea $(B r g+B r f)$ y $B a$, la cual es $11 \%, 40 \%$ y $56 \%$ a 1050,2380 y $3060 \mathrm{~m}$ de altitud (Leuschner \& Moser, 2008). Dicha relación en los bosques de San Pedro,
Antioquia, fue $83 \%$. De hecho dicha relación es aún mayor en rastrojos (216\%), lo cual sugiere que en coberturas dominadas por árboles pequeños y arbustos hay mayor asignación energética a la biomasa subterránea. Asimismo, no se encontraron referencias del cambio en la asignación energética entre los compartimientos arbóreos respecto al diámetro o avance del proceso sucesional en bosques montano bajos. Sin embargo, la relación entre las ecuaciones de biomasa de árboles individuales ( $\mathrm{brg}$ y $b a$ ) de Porce en función del $D$ se comporta como una exponencial negativa que inicia en $50 \%$ y cae asintóticamente a 18\% (del Valle et al., 2011), y con el promedio de las biomasas de rastrojos de tres años de edad en Porce se puede deducir que Brg/Ba es 30\% (del Valle et al., 2011). También se ha encontrado que $\mathrm{Brg} / \mathrm{Ba}$ cambia con respecto a la edad del proceso sucesional. Dicha relación alcanza $25 \%$ a los 4 años, luego cae hasta $18 \%$ a los 25 años, para luego tender a una asíntota de $27 \%$ (del Valle et al., 2011). De acuerdo con los patrones analizados para los bosques secundarios en diferentes estados sucesionales ubicados en zonas de montaña, dichas relaciones podrían ser mayores debido a la inversión de la asignación energética entre $B a$ y la biomasa subterránea.

Una aproximación que puede ayudar a entender las diferencias en asignación energética es el estudio de la distribución de la biomasa respecto al diámetro. En la presente investigación se encontró que la biomasa de bosques tiene una distribución unimodal respecto a los diámetros, mientras que otra investigación sugiere que dicha distribución es en forma de J, con incremento de la biomasa proporcional al diámetro (Culmsee et al., 2010). Sin embargo, no se encontraron investigaciones que usaran dichas distribuciones de manera desagregada para la $B a, B r g$ y $B r f y$ que permitieran comparar los resultados obtenidos.

Otros componentes desempeñan un importante papel en la estructura de los bosques montanos tales como lianas (Gehring et al., 2004) y epífitas (Walker \& Ataroff, 2002; Gradstein, 2008). Por ejemplo, la densidad y área basal de lianas encontradas en esta investigación son muy similares a 410 lianas ha ${ }^{-1}$ y $0.5 \mathrm{~m}^{2}$ ha $^{-1}$ de Colombia (van der Heijden \& 


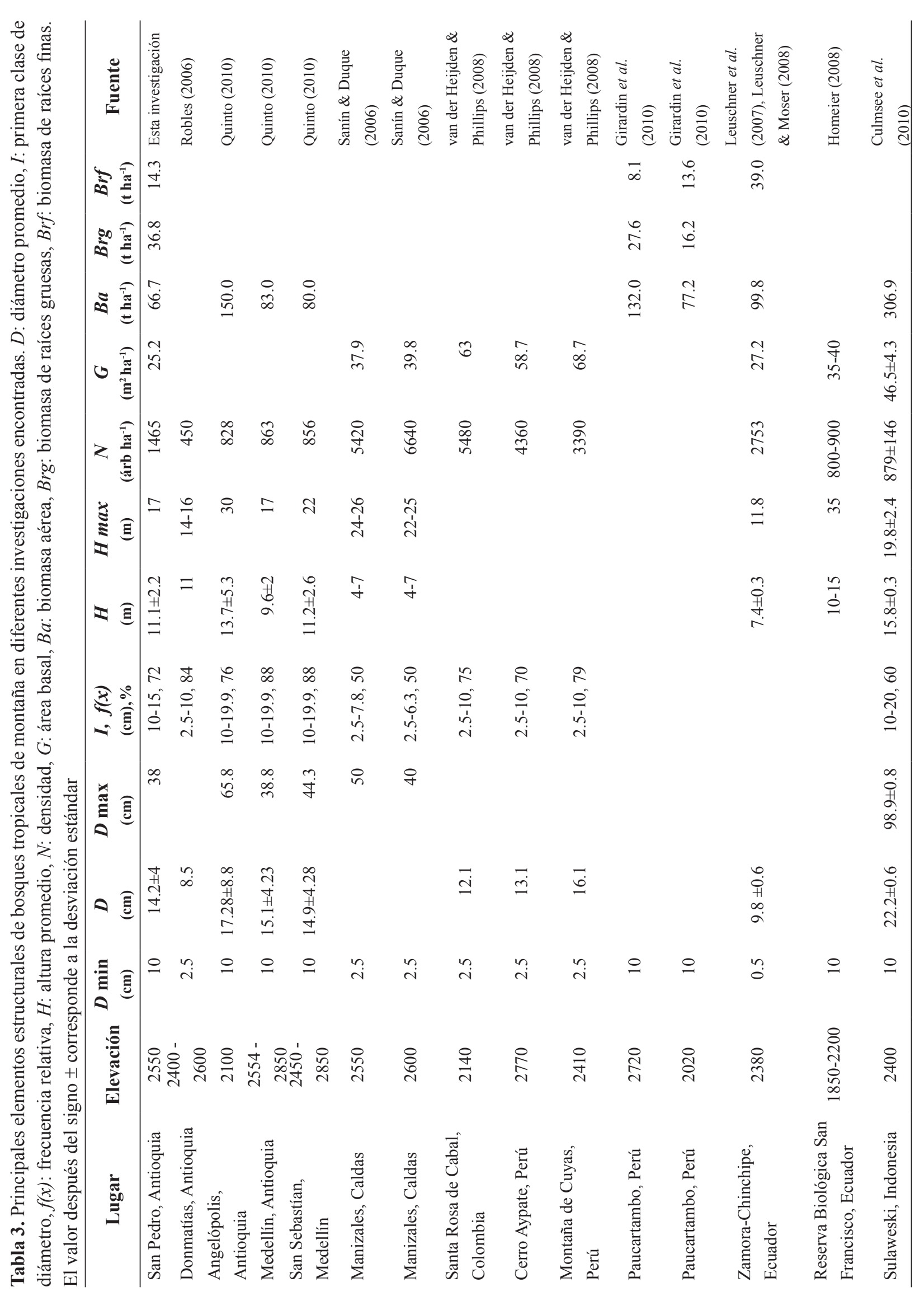


Phillips, 2008), 700-850 lianas ha-1 y $0.2-0.65 \mathrm{~m}^{2}$ ha $^{-1}$ de Ecuador (Homeier, 2008) y 380-600 lianas ha $^{-1}$ y $0.3-0.8 \mathrm{~m}^{2}$ ha $^{-1}$ de Perú (van der Heijden \& Phillips, 2008). La proporción del área basal de lianas respecto al área basal de árboles fue $1.3 \%$; dato similar al reportado para bosques húmedos (Hegarty \& Caballe, 1991). Igualmente, no se encontraron referencias de biomasa de lianas para bosques montanos tropicales. Sin embargo, la proporción de $B l$ respecto a $B a$ fue $6.2 \%$, la cual está en el rango 1.1 y $14 \%$ reportado por otros autores (Gerwing \& Farias, 2000; Orrego \& del Valle, 2001; Gehring et al., 2004).

Por otra parte, otro de los principales rasgos del bosque húmedo montano es la abundancia de epífitas. Estas son plantas arbóreas tales como orquídeas, bromelias, aráceas, ericáceas, piperáceas, cactus, helechos, briofitas, líquenes, entre otras. (Gradstein, 2008). En la presente investigación se encontró que la presencia de epífitas es entre media y alta, similar a la encontrada en Colombia en los municipios de Donmatías (Robles, 2006) y Manizales (Sanín \& Duque, 2006). Además, su biomasa no es nada despreciable si se compara con la biomasa arbórea aérea, pues se estima que está entre 12 y $44 \mathrm{t} \mathrm{ha}^{-1}$ en bosques de igual elevación en Colombia y Venezuela (Wolf, 1993; Walker \& Ataroff, 2002; Hsu et al., 2002; Gradstein, 2008).

Con el crecimiento del bosque, algunos árboles expanden sus copas horizontalmente, con esto logran interceptar la mayor cantidad de luz que penetra al bosque y, consecuentemente, alcanzan las mayores tallas. Esta constante competencia entre los individuos del bosque hace que los árboles de tallas inferiores cercanos a los dominantes vean afectado su crecimiento por la supresión que experimentan y eventualmente mueran, este fenómeno es conocido como raleo natural o autorraleo (Oliver \& Larson, 1990). Así, la materia orgánica correspondiente a árboles muertos en pie fue muy similar al promedio de bosques primarios de Porce, estimada en 2.6 tha-1 (Orrego \& del Valle, 2001). Aun así, no se encontraron referencias que permitieran comparar la necromasa de los árboles muertos en pie de bosques montanos.

\section{CONCLUSIONES}

Se caracterizó la estructura de bosques montanos tropicales mediante el análisis de tallas promedio, distribuciones diamétricas y la estimación de biomasa y necromasa contenida en los árboles muertos en pie. Las distribuciones diamétricas en los bosques y rastrojos en el norte de Antioquia exhibieron una forma de $J$-invertida, caracterizada por un número alto de individuos de tallas pequeñas y muy pocos de tallas grandes. Asimismo, el modelo Hugershoff fue mucho más flexible que las tradicionales funciones de distribución de probabilidades en el ajuste estadístico de las distribuciones diamétricas de bosques y rastrojos en la zona del norte de Antioquia.

Las tallas medias de los individuos y la biomasa estimada fueron similares o ligeramente inferiores a las encontradas en la misma franja altitudinal en otros países. Sin embargo, los bosques montanos tropicales tienen características estructurales diferentes a los bosques tropicales de tierras bajas. Las diferencias básicas entre estos dos ecosistemas son la disminución en las tallas de los individuos, reducción de la biomasa arbórea aérea e inversión en la asignación energética entre los compartimientos de la biomasa arbórea, aspectos poco estudiados hasta el momento.

\section{AGRADECIMIENTOS}

La presente investigación hizo parte de los proyectos Valoración Económica, Ecológica y SocioCultural de Bienes y Servicios Ambientales en la Cuenca del Río Grande, contrato 8811, y Diseño de un Modelo Conceptual de Análisis de Riesgo Asociado con Cambios Ambientales Globales en la Cuenca del Río Grande Caso de Aplicación: Estudio de Impactos Proyectados para Diferentes Escenarios de Cambio Ambiental en la Cuenca del Río Hato, con Énfasis en la Disponibilidad y Calidad del Recurso Hídrico, contrato 8824 . Los proyectos se ejecutaron por la Universidad Nacional de Colombia, Sede Medellín, y la Universidad de Antioquia, y fueron financiados por la Corporación Autónoma Regional del Centro 
de Antioquia, Corantioquia. Agradecimientos a Cristian David Ramírez, Angélica Liliana Ochoa y Mario Alexander Hoyos por su colaboración en el establecimiento de las parcelas permanentes.

\section{REFERENCIAS BIBLIOGRÁFICAS}

Achard, F., Eva, H., Stibig, H-J., Mayaux, P., Gallego, J., Richards, T., \& Malingreau, J-P.(2002). Determinations of deforestaion rates of the world's humid tropical forest. Science, 297, 999-1002.

Aiba, S.-i., Kitayama, K., \& Takyu, M. (2004). Habitat associations with topography and canopy structure of tree species in a tropical montane forest on Mount Kinabalu, Borneo. Plant Ecology, 174, 147-161.

Akindele, O.S., \& Onyekwelu, J.C. (2011). Review: Silviculture in Secundary Forest. En: S. Günter, M. Weber, B. Stimm \& R. Mosandl (eds.). Silviculture in the tropics (pp. 351-368.). Muenchen: Springer.

Álvarez-Yépiz, J.C., Martínez-Yrízar, A., Búrquez, A., \& Lindquist, C. (2008). Variation in vegetation structure and soil properties related to land use history of old-growth and secondary tropical dry forests in northwestern Mexico. Forest Ecology and Management, 256, 355-366.

Álvarez, E., Duque, A., Saldarriaga, J., Cabrera, K., de las Salas, G., del Valle, J.I., Lema, A., Moreno, F., Orrego, S.A., \& Rodríguez, L. (2012). Tree above-ground biomass allometries for carbon stocks estimation in the natural forests of Colombia. Forest Ecology and Management, 267, 297-308.

Alves, L.F., Vieira, S.A., Scaranello, M.A., Camargo, P.B., Santos, F., Joly, C., \& Martinelli, L.A. (2010). Forest structure and live aboveground biomass variation along an elevational gradient of tropical Atlantic moist forest (Brazil). Forest Ecology and Management, 260, 679-691.

Ariza, W., Toro, J.L., \& Lores, A. (2009). Análisis florístico y estructural de los bosques premontanos en el municipio de Amalfi (Antioquia, Colombia). Colombia Forestal, 12, 81-102.

Armenteras, D., Gast, F., \& Villareal, H. (2003). Andean forest fragmentation and the representativeness of protected natural areas in the eastern Andes, Colombia. Biological Conservation, 113, 245-256.

Armenteras, D., Rodríguez, N., Retana, J., \& Morales, M. (2011). Understanding deforestation in montane and lowland forests of the Colombian Andes. Regional Environmental Change, 11, 693-705.

Bolivar, J., Buitrago, M.F., \& Cuartas, L.F. (2006). Ecuaciones de conicidad, volumen y biomasa para bosques de zonas altas del valle de San Nicolás, Colombia (Informe de campamento Ingeniería Forestal). Medellin: Universidad Nacional de Colombia. $45 \mathrm{p}$.

Brown, S. (1997). Estimating biomass and biomass change of tropical forest: a primer. FAO Forestry Paper 134. Roma: FAO. Recuperado de http://www.fao.org/docrpe/ w4095E00.htm.

Brown, S. \& Lugo, A.E. (1990). Tropical secondary forests. Journal of Tropical Ecology, 6, 1-32.

Bush, M.B., Hanselman, J.A., \& Hooghiemstra, H. (2011). Andean montane forest and climate change. En: M.B. Bush, J.R. Flenley \& W. Gosling (eds.). Tropical rainforest responses to climatic change (pp. 35-60). Milton Keynes: Springer. 
Canavos, G. (1988). Probabilidad y estadística: Aplicaciones y métodos. Mexico: McGraw Hill. $651 \mathrm{p}$.

Chacón, P., Leblanc, H.A., \& Russo, R.O. (2007). Fijación de carbono en un bosque secundario de la región tropical húmeda de Costa Rica. Tierra Tropical, 3, 1-11.

Chazdon, R.L., Peres, C.A., Dent, D., Sheil, D., Lugo, A.E., Lamb, D., Storck, N.E., \& Miller, S.E. (2009). The potential for species conservation in tropical secondary forest. Conservation Biology, 23, 1406-1417.

Chokkalingam, U., de Jong, W., Smith, J., \& Sabogal, C. (2001). Secondary forest in Asia: Their diversity, importance, and role in future environmental management. Journal of Tropical Forest Science, 13(4), 5-20.

Clark, D.B., \& Clark, D.A. (2000). Landscapescale variation in forest structure and biomass in a tropical rain forest. Forest Ecology and Management, 137, 185-198.

Cook, E., Briffa, K., Shiyatov, S., Mazepa, V., \& Jones, P.D. (1992). Data analysis. En: E. Cook \& L. Kairiukstis (eds.). Methods of dendrochronology: applications in the environmental sciences (pp. 97-162). Dordrecht: Kluwer Academic Publishers.

Crausbay, S.D., \& Hotchkiss, S.C. (2010). Strong relationships between vegetation and two perpendicular climate gradients high on a tropical mountain in Hawai'i. Journal of Biogeography, 37, 1160-1174.

Crawley, M. (2007). The R book. Mississauga: John Wiley \& Sons, Ltd. 951 p.

Culmsee, H., Leuschner, C., Moser, G., \& Pitopang, R. (2010). Forest aboverground biomass along and elevational transect in Sulaweski, Indonesia, and the role of Fagaceae in tropical montane rain forest. Journal of Biogeography, 37, 960-974.
Cummings, D.L., Kauffman, J.B., Perry, D.A., \& Hughes, R.F. (2002). Aboverground biomass and structure of rainforests in the southwestern Brazilian Amazon. Forest Ecology and Management, 163, 293-307.

Daumenge, C., Gilmor, D., Ruíz Pérez, M., \& Blockhus, J. (1995). Tropical montane cloud forest: Conservation status and management issues. En: L.S. Hamilton, J.O. Juvik \& F.N. Scatena (eds.). Tropical montane cluod forest. Proceedings of an international symposium on ecological studies (pp. 24-37). New York: SpringerVerlag.

de las Salas, G. (2002). Los bosques secundarios de América tropical: prespectivas para su manejo sostenible. Bois et Forêts des Tropiques, 272, 63-73.

del Valle, J.I., Restrepo, H.I., Londoño, M.M. (2011). Recuperación de la biomasa mendiante la sucesión secundaria, Cordillera Central de los Andes, Colombia. Revista de Biología Tropical, 59, 13371358.

Fehse, J., Hofstede, R., Aguirre, N., Paladines, C., Kooijman, A., \& Sevink, J. (2002). High altitude tropical secondary forest: a competitive carbon sink?. Forest Ecology and Management, 163, 9-25.

Fukushima, M., Kanzaki, M., \& Hara, T. (2008). Secondary forest succession after the cessation of swidden cultivation in the montane forest area in Northern Thailand. Forest Ecology and Management, 255, 1994-2006.

Gehring, C., Park, S., \& Denich, M. (2004). Liana allometric biomass equations for Amazonian primary and secundary forest. Forest Ecology and Management, 195, 6983. 
Gerwing, J.J., \& Farias, D.L. (2000). Integrating liana abundance and forest stature into an estimate of total aboverground biomass for an eastern Amazonian forest. Journal of Tropical Ecology, 16, 327-335.

Girardin, C.A., Malhi, Y., Aragão, L.E., Mamani, M., Huaraca, W., Durand, L., Feeley, K.J., Rapp, J., Silva-Espejo, J.E., Silmans, M., Salinas, N., \& Whittaker, R.J. (2010). Net primary productivity allocation an cycling of carbon along a tropical forest elevational transect in the Peruvian Andes. Global Change Biology, 16, 3176-3192.

Gradstein, S.R. (2008). Epiphytes of tropical montane forest-impact of deforastion and climate change. En: S.R. Grandstein, J. Homeier \& D. Gansert (eds.). The tropical montane forest. Patterns and processes in a biodiversity hotspot (pp. 51-66). Göttingen: Göttingen Centre for Biodiversity and Ecology, Universitätsverlag Göttingen.

Guariguata, M.R., \& Ostertag, R. (2001). Neotropical secondary forest succession: changes in structural and functional characteristics. Forest Ecology and Management, 148, 185-206.

Hegarty, E.E., \& Caballe, G. (1991). Distribution and abundance of vines in forest communities. En: F.E. Putz \& H.A. Mooney (eds.). The biology of vines (pp. 263-282). Cambridge: Cambridge University Press.

Hering, K.G. (2003). A scientific formulation of tropical forest management. Ecological Modelling, 166, 211-238.

Hiroaki, I., \& McDowell, N. (2002). Age-related development of crown structure in coastal Douglas-fir trees. Forest Ecology and Management, 169, 257-270.
Hölscher, D. (2008). Hydrology of natural and anthropogenically altered tropical montane rainforests with special reference to rainfall interception. En: S.R. Grandstein, J. Homeier \& D. Gansert (eds.). The tropical montane forest. Patterns and processes in a biodiversity hotspot (pp. 129-138). Göttingen: Göttingen Centre for Biodiversity and Ecology, Universitätsverlag Göttingen.

Homeier, J. (2008). The influence of topography on forest structure and regeneration dynamics in an Ecuadorian montane forest. En: S.R. Grandstein, J. Homeier \& D. Gansert (eds.). The tropical montane forest. Patterns and processes in a biodiversity hotspot (pp. 97-108). Göttingen: Göttingen Centre for Biodiversity and Ecology, Universitätsverlag Göttingen.

Hsu, C.C., Horng, F.W., \& Kuo, C.M. (2002). Epiphyte biomass and nutrient capital of a moist subtropical forest in north-eastern Taiwan. Journal of Tropical Ecology, 18, 659-670.

Kangas, A. (2006). Mensurational aspects. En: A. Kanagas \& M. Maltamo (eds.). Forest Inventory: Methodology and Applications (pp. 53-64). Dordrecht: Springer.

Kessler, M. \& Kluge, J. (2008). Diversity and endemism in tropical montane forest - from patterns to process. En: S.R. Grandstein, J. Homeier \& D. Gansert (eds.). The tropical montane forest. Patterns and processes in a biodiversity hotspot (pp. 35-50). Göttingen: Göttingen Centre for Biodiversity and Ecology, Universitätsverlag Göttingen.

Lamprecht, H. (1990). Silvicultura en los trópicos. Los ecosistemas forestales en los bosques tropicales y sus especies arbóreas. Posiblidades y métodos para un aprovechamiento sostenido. Eschborn: GTZ (GMBH). 335 p. 
Leuschner, C., Moser, G., Bertsch, C., Röderstein, M., \& Hertel, D. (2007). Large altitudinal increase in tree root/shoot ratio in tropical mountain forest of Ecuador. Basic and Applied Ecology, 8, 219-230.

Leuschner, C., \& Moser, G. (2008). Carbon allocation and productivity in tropical mountain forests. En: S.R. Grandstein, J. Homeier \& D. Gansert (eds.). The tropical montane forest. Patterns and processes in a biodiversity hotspot (pp. 109-128). Göttingen Centre for Biodiversity and Ecology, Universitätsverlag Göttingen.

Mittermeier, R.A., Turner, W.R., Larsen, F.W., Brooks, T.M., \& Gascon, C. (2011). Global biodiversity conservation: the critical role of hotspots. En: F. Zachos \& J.C. Habel (eds.). Biodiversity hotspots: Distribution and protection of conservation priority areas (pp. 3-22). New York: Springer.

Myers, N., Mittermeier, R.A., Mittermeier, C.G., da Fonseca, G., \& Kent, J. (2000). Biodiversity hotspots for conservation priorities. Nature, 403, 853-858.

Neeff, T., \& dos Santos, J.R. (2005). A growth model for secondary forest in Central Amazonia. Forest Ecology and Management, 216, 270-282.

Newton, A.C. (2007). Forest Ecology and Conservation: a handbook of techniques. New York: Oxford University Press. 454 p.

Ochoa-Gaona, S., González-Espinosa, M., Meave, J.A., \& Sorani-Dal Bon, V. (2004). Effect of forest fragmentation on the woody flora of the highlands of Chiapas, Mexico. Biodiversity and Conservation, 13, 867-884.

Oliver, C.D., \& Larson, B.C. (1990). Forest stand dynamics. New York: McGraw-Hill Inc. $419 \mathrm{p}$.
Orrego, S.A., \& del Valle, J.I. (2001). Existencias y tasa de incremento neto de la biomasa $\mathrm{y}$ del carbono en bosques primarios $\mathrm{y}$ secundarios de Colombia. Valdivia: Simposio Internacional Medición y Monitoreo de la Captura de Carbono en Ecosistemas Forestales. 31 p.

Parresol, B.R. (2002). Biomass. En: A.H. ElShaarawi \& W.W. Piegorsch (eds.). Encyclopedia of Environmetrics (pp. 196198). New York: John Wiley \& Sons, Ltd.

Prodan, M. (1968). Forest biometrics. Oxford: Pergamon Press Ltd. 447 p.

Quinto, H. (2010). Dinámica de la biomasa aérea en bosques primarios de Colombia y su relación con la precipitación y la altitud (Tesis de Maestría en Bosques y Conservación Ambiental). Medellín: Universidad Nacional de Colombia, sede Medellín. 75 p.

R Development Core Team. (2010). R: A language and environment for statistical computing. Vienna: R Foundation for Statistical Computing. ISBN: 3-900051-07-0, recuperado de http://www.R-project.org.

Richards, K.R., \& Stokes, C. (2004). A review of forest carbon sequestration cost studies: a dozen years of reserch. Climatic Change, 63: 1-48.

Richter, M. (2008). Tropical mountain forest - distribution and general features. En: S.R. Grandstein, J. Homeier \& D. Gansert (eds.). The tropical montane forest. Patterns and processes in a biodiversity hotspot (pp. 7-24). Göttingen: Göttingen Centre for Biodiversity and Ecology, Universitätsverlag Göttingen.

Robles, C. (2006). Caracterización de la diversidad y uso de la flora silvestre en el municipio de Donmatías - Antioquia. Medellín: Corantioquia. $76 \mathrm{p}$. 
Rollet, B. (1980). Organización. En UNESCO, UNEP, FAO (eds.). Ecosistemas de los bosques tropicales: Informe sobre el estado de conocimientos (pp.: 126-154). Madrid: UNESCO, UNEP, FAO.

Saldarriaga, J.G. (1994). Recuperación de la selva de "tierra firme" en el alto río Negro, Amazonía colombiana-venezolana. Bogotá: Tropenbos. 201 p.

Sanín, D., \& Duque, C. (2006). Estructura y composición florística de dos transectos localizados en la reserva forestal protectora Río Blaco (Manizales, Caldas, Colombia). Museo de Historia Natural, 10, 45-75.

Schlegel, B., Gayoso, J., \& Guerra, J. (2001). Manual de procedimientos para inventarios de carbono en ecosistemas forestales. Valdivia: Universidad Austral de Chile. 16 p.

Schwarzkopf, T., Riha, S., Fahey, T., \& Degloria, S. (2011). Are cloud forest tree structure and environmental related in the velezuelan Andes. Austral Ecology, 36, 280-289.

Sierra, C., del Valle, J.I., \& Orrego, S.A. (2001). Ecuaciones de biomasa de raíces y sus tasas de acumulación en bosques sucesionales y maduros tropicales de Colombia. Valdivia: Simposio Internacional Medición y Monitoreo de la Captura de Carbono en Ecosistemas Forestales. 16 p.

Sierra, C., del Valle, J.I., Orrego, S.A., Moreno, F., Harmon, M., Zapata, M., Colorado, G., Herrera, M.A., Lara, W., Restrepo, D.E., Berrouet, L.M., Loaiza, L.M., \& Benjumea, J.F. (2007). Total carbon stocks in a tropical forest landscape of the Porce region, Colombia. Forest Ecology and Management, 243, 299-309.
Toledo, M., Salick, J., Liselle, B., \& Jørgensen, P. (2005). Composición florística y usos de bosques secundarios en la provincia de Guarayos, Santa Cruz, Bolivia. Revista Bolivariana de Ecología y Conservación, 18, 1-16.

Uhl, C., Buschbacher, R., \& Serrão, E.A.S. (1988). Abandoned pastures in eastern Amazonia. I. Patterns of plant succession. Journal of Ecology, 76, 663-681.

van der Heijden, G.M.F., \& Phillips, O. (2008). What controls liana success in Netropical forest? Global Ecology and Biogeography, 17, 372-383.

Vilches, B., Chazdon, R.L., \& Milla, V. (2008). Dinámica de la regeneración natural en cuatro bosques secundarios tropicales de la región de Huertar Norte, Costa Rica: su valor para la conservación o uso comercial. Recursos Naturales y Ambiente, 55: 118128.

Walker, R., \& Ataroff, M. (2002). Biomasa epifita y su contenido de nutrientes en una selva nublada andina, Venezuela. Ecotrópicos, 15, 203-210.

Wolf, J.H.D. (1993). Diversity patterns and biomass of epiphytic bryophytes and lichens along an altitudinal gradient in the northern Andes. Annals of the Missouri Botanical Garden, 80, 928-960.

Whitmore, T.C. (1998). An introduction to tropical rain forest. Clarendon: Oxford University Press. 282 p.

Yepes, A.P., del Valle, J.I., Jaramillo, S.L., \& Orrego, S.A. (2010). Recupueración estructural en bosques succesionales andinos de Porce (Antioquia, Colombia). Revista de Biología Tropical, 58, 427-445. 